\title{
Further Studies of Electron Avalanche Gain in Liquid Argon
}

\author{
J.G. Kim, S. M. Dardin, R.W. Kadel, J.A. Kadyk, K.H. Jackson, V. Peskov, W.A. Wenzel, K.S. Joo
}

\begin{abstract}
Previously [1] we showed how small admixtures of xenon (Xe) stabilize electron avalanches in liquid Argon (LAr). In the present work, we have measured the positive charge carrier mobility in LAr with small admixtures of Xe to be $6.4 \times 10^{-3}$ $\mathrm{cm}^{2} / \mathrm{Vsec}$, in approximate agreement with the mobility measured in pure LAr, and consistent with holes as charge carriers. We have measured the concentration of Xe actually dissolved in the liquid and compared the results with expectations based on the amount of Xe gas added to the LAr. We also have tested LAr doped with krypton to investigate the mechanism of avalanche stabilization.
\end{abstract}

\section{INTRODUCTION}

I $\mathrm{n}$ our previous paper [1], we showed how small admixtures of Xe could stabilize electron avalanches in liquid argon (LAr). The avalanches were produced in a high electric field at the tip of a sharp needle, irradiated by $60 \mathrm{keV}$ photons from an ${ }^{241} \mathrm{Am}$ source. We observed two sets of pulses: one set with signal amplitude independent of the applied pressure, and a second set whose amplitude depended on the pressure. Subsequently, and reported in this paper, we made several measurements to verify our understanding of the electrostatics of our apparatus, have investigated the dependence of current versus applied voltage for sources with two different gamma-ray energies and fluxes, and have

Manuscript received Dec 2, 2002. This work was supported by the Director, Office of the Energy Research of the U.S. Department of Energy under Contract No. DE-AC03-76SF00098.

J. G. Kim is with the Physics Division, Lawrence Berkeley National Laboratory, Berkeley, CA 94720 USA on leave from Physics Department, MyongJi University, Young-In, Korea 449-728 (telephone: 510-486-4579, email: JGKim@lbl.gov).

S. M. Dardin is with the Physics Division, Lawrence Berkeley National Laboratory, Berkeley, CA 94720 USA (telephone: 510-486-6598, e-mail: SMDardin@lbl.gov).

K. H. Jackson is with the Material Science Division, Lawrence Berkeley National Laboratory, Berkeley, CA 94720 USA (telephone: 510-486-6894, e-mail: KHJackson@lbl.gov).

R. W. Kadel is with the Physics Division, Lawrence Berkeley National Laboratory, Berkeley, CA 94720 USA (telephone: 510-486-7360, e-mail: RWKadel@lbl.gov).

J. A. Kadyk is with the Physics Division, Lawrence Berkeley National Laboratory, Berkeley, CA 94720 USA (telephone: 510-486-7189, e-mail: JAKadyk@lbl.gov).

V. Peskov is with the Royal Institute of Technology, Stockholm, Sweden (telephone: 48-8-5537-8182, e-mail: Vladimir.Peskov@cern.ch ).

W. A. Wenzel is with the Physics Division, Lawrence Berkeley National Laboratory, Berkeley, CA 94720 USA (telephone: 510-486-6918, e-mail: B_Wenzel@lbl.gov).

K.S. Joo is with Physics Department, MyongJi University, Young-In, Korea 449-728 (telephone: 82-31-330-6167, e-mail: ksjoo@mju.ac.kr). measured and compared the corresponding pulse height spectra. For a better understanding of the energy transfer mechanism in electron avalanche processes in LAr, various concentrations of krypton $(\mathrm{Kr})$ have been added, and the results compared to those obtained in similar tests using Xe.

\section{EXPERIMENTAL DETAIL}

\section{A. Description of the Test Vessel and Detector}

The experimental setup has been previously described in [1], and schematics of the apparatus are shown in Figs. 1 and 2 . The detector consists of a chemically sharpened tungsten needle (anode), the tip of which was placed midway between two conducting planes: the lower plane (ground plane) and the needle are at zero DC potential, and the upper plane, the drift electrode (cathode), is at negative HV (Fig. 2). The detector is enclosed in a stainless steel "test vessel" that can be baked out and evacuated. The test vessel is a cylinder of about 6 liter total volume filled with $\sim 3$ liters of LAr for testing. It has several ports for: (a) a moveable $0.1 \mathrm{Ci}^{241} \mathrm{Am}$ source internal to the test vessel that illuminates the LAr with gamma rays of peak energy $60 \mathrm{keV} \mathrm{[2];} \mathrm{(b)} \mathrm{a} \mathrm{thin} \mathrm{aluminum}$ window $\left(0.25 \mathrm{~mm}\right.$ thick) allowing use of an external ${ }^{57} \mathrm{Co}$ source of $\sim 5 \mathrm{mCi}$ strength, and (c) for electrical leads: anode, cathode, temp, etc. To achieve the purity level needed to drift electrons, the boil-off gas from the LAr supply dewar (99.99\% pure) is passed through a series of three filters: a 4A molecular sieve [3], an "Oxysorb" $\mathrm{O}_{2}$ filter [4], and a "Gatekeeper" inert gas purifier including a $0.5 \mu \mathrm{m}$ particulate filter [5]. After filtering, the Ar gas to be condensed for our tests has a measured oxygen concentration less than $\sim 10 \mathrm{ppb}$ (the minimum sensitivity of our $\mathrm{O}_{2}$ meter); the filter specifications [5] indicate that this concentration should not exceed $1 \mathrm{ppb}$. The desired concentration of $\mathrm{Xe}$ was determined by adding to the LAr, a calculated volume of the gas mixture $\mathrm{Ar} / \mathrm{Xe}(95 \% / 5 \%)$ using a mass flow meter. We pressurize the system between zero and $2.6 \mathrm{~atm}$ (gauge pressure) using Helium gas. 


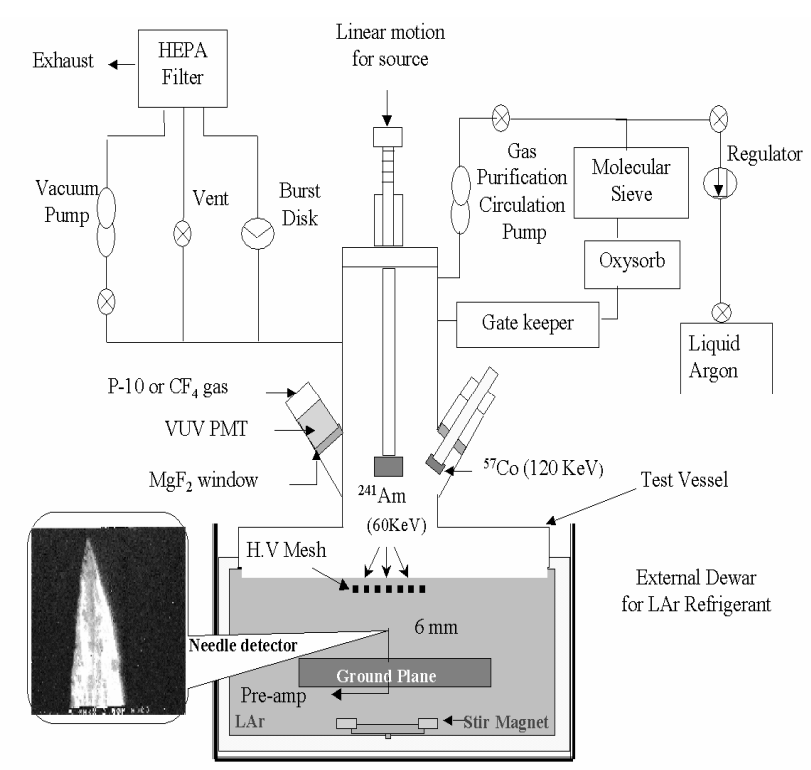

Fig. 1. A schematic of the test vessel in which avalanche tests were done using liquid argon, and a photograph of a needle tip using an electron microscope. The He pressurization gas source is not indicated. Measurement of anode current and pulses is done by means of electrical connections on one port

\section{B. Mixing and Sampling Technique}

Previously, we implemented a mixing system using a pivoted alnico bar magnet inside the test vessel, rotated under the influence of an external driving magnet [1]. Subsequently, we have improved our mixing system and added a sampling system that extracts a $\sim 10 \mathrm{ml}$ sample of the $\mathrm{LAr} / \mathrm{Xe}$ mixture for analysis. By heating, this liquid sample is expanded to the gaseous state into a one-liter sampling bottle that can be shipped to an analysis laboratory. As indicated in our previous paper[1], a systematic decrease in the pulse height occurs over a run of several hours, which may be the result of the variation of impurtity concentrations in the sample with time.

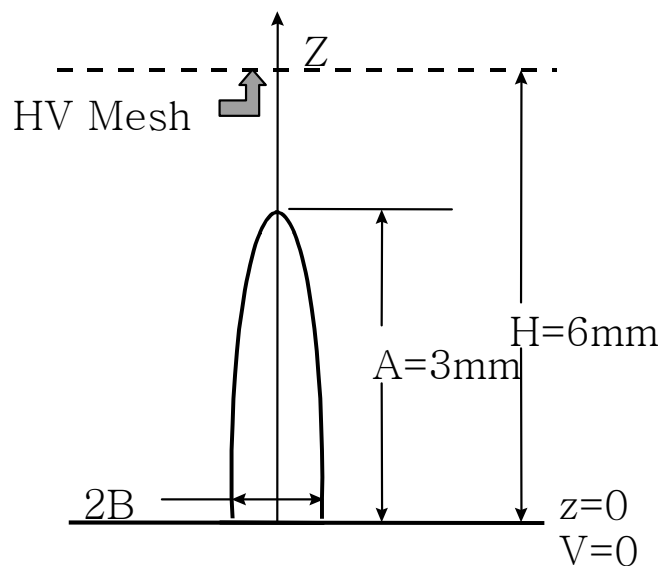

Fig. 2. Needle geometry schematic. The horizontal scale has been stretched for clarity. The radius of the needle at the base is $\sim 127 \mu \mathrm{m}$, and the radius at the tip is $\sim 0.25 \mu \mathrm{m}$. $\mathrm{H}$ and A are typically $6 \mathrm{~mm}$ and $3 \mathrm{~mm}$, respectively.

\section{RESULTS AND DISCUSSION}

\section{A. Positive Charge Carrier Mobility}

In our previous paper [1] we presented measurements of the needle current versus voltage for avalanches in pure LAr, and suggested a model to explain the behavior. We have since repeated that test in LAr doped with $\sim 25 \mathrm{ppm}$ Xe for two different radioactive sources. The results are shown in Fig. 3, along with the previous measurement. The newer data include three sets of measurements: 1) a ${ }^{241} \mathrm{Am}$ source (60 $\mathrm{keV}$ photons) at $\sim 15 \mathrm{~cm}$ from the tip of the needle; 2) the ${ }^{241} \mathrm{Am}$ source at $\sim 7.5 \mathrm{~cm}$ from the needle; and 3) a ${ }^{57} \mathrm{Co}$ source (122 keV photons) at $\sim 7.5 \mathrm{~cm}$ from the needle. At lower voltages, the needle current induced by the ${ }^{57} \mathrm{Co}$ source is about a factor of 10 larger than for the ${ }^{241} \mathrm{Am}$ source when both are $7.5 \mathrm{~cm}$ away from the needle. This is roughly consistent with our expectations, based upon the relative source strengths, the difference in geometries in the two cases, and the difference in photon energies and corresponding absorption lengths. The measured rates of pulses from the two sources are also explained by these differences. However, above about $1 \mathrm{nA}$ (see Fig. 3), all of the data points at the same voltage nearly coincide. Our previous data with pure Ar lie consistently above these newer points, which we believe comes from the previously smaller distance between the needle tip and the drift electrode $(\sim 2 \mathrm{~mm})$ as compared to the newer data $(\sim 3 \mathrm{~mm})$. These data suggest that the current at higher fields is independent of the source intensity and photon energy, and is, therefore, space charge limited.

In the situation where the mobility of the charge carriers is independent of the electric field, a significant space charge

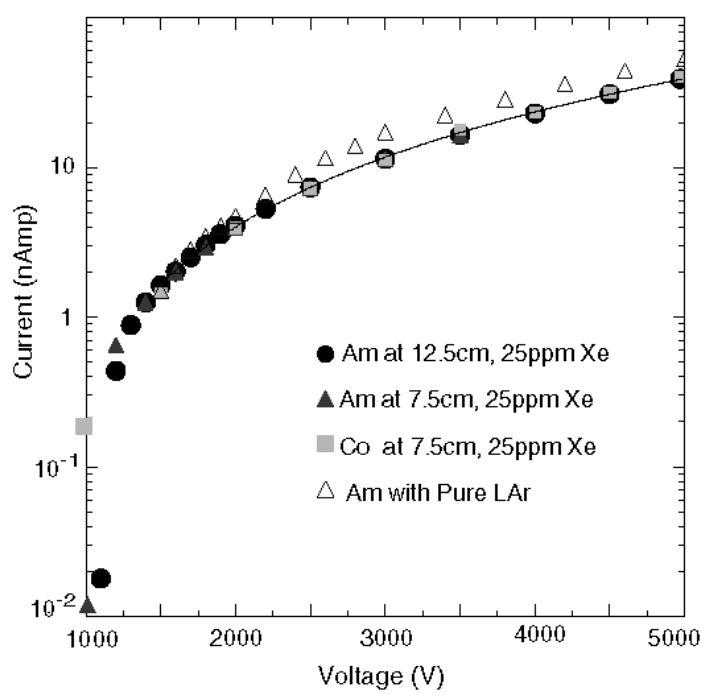

Fig. 3. Current vs. voltage for pure LAr and LAr with $25 \mathrm{ppm}$ Xe. Fit to the black solid points is shown. (See text)

density develops near the needle tip. The current versus voltage relationship has been calculated for the case of field emission from a needle [6] to be $V=V_{v a c}+2\left(8 d / 3 \alpha \varepsilon \mu^{+}\right)^{1 / 2} i^{1 / 2}$. Here, $d$ is the distance from the tip of the needle to the screen ( $2 \mathrm{~mm}$ or $3 \mathrm{~mm}), \varepsilon$ is the dielectric constant of $\operatorname{LAr}\left(1.51 \varepsilon_{o}\right.$, 
$\varepsilon_{0}=$ vacuum permittivity), $\mu^{+}$is the positive charge carrier mobility, $\alpha$ is a constant equal to 0.6 in the point/sphere geometry approximation, and $V_{v a c}$ is the voltage in vacuo necessary to produce the emitted current, and is determined by fitting the above expression to our data. This equation is written for the case of field emission from a cathode needle (hence current in vacuo), but it is expected to apply for our tests as well, since in both instances the current-voltage dependence is dominated by space charge limitations. If we use $\mathrm{d}=3 \mathrm{~mm}$ for our new data, we find that the positive charge carrier mobility, $\mu^{+}$, is $6.4 \times 10^{-3} \mathrm{~cm}^{2} / \mathrm{Vsec}$. This is in reasonable agreement with the value that we obtain by fitting our earlier data using pure LAr: $7.0 \times 10^{-3} \mathrm{~cm}^{2} /$ Vsec. These measurements are in approximate agreement with newer published values of the mobility of positive charge carriers in pure $\operatorname{LAr}\left(9.4 \times 10^{-3} \mathrm{~cm}^{2} / \mathrm{Vsec}\right.$ [7]), but disagree by an order of magnitude with older published values $\left(\sim 6 \times 10^{-4}[8]\right)$. The authors of [9] conclude that for very pure LXe, the positive charge carriers are principally holes and not positive ions, because the high positive carrier mobility in LXe $\left(3.7 \times 10^{-3}\right.$ $\left.\mathrm{cm}^{2} / \mathrm{Vsec}\right)$ is inconsistent with the measured viscosity of the liquid and the hard-core radius of the $\mathrm{Xe}$ ions. This is also the case for Ar ions, as we have verified by a similar calculation for viscosity of Xe ions in LAr.Our results lead us to a similar conclusion: the mobility for pure LAr with small admixtures of $\mathrm{Xe}$ is consistent only with holes as the primary positive charge carrier and not consistent with Xe or Ar ions.

\section{B. Pulse Height Saturation vs. Photon Energy}

We have measured the pulse height spectra for the ${ }^{241} \mathrm{Am}$ source at $7.5 \mathrm{~cm}$ from the needle, and the spectrum obtained when both the ${ }^{241} \mathrm{Am}$ source and the ${ }^{57} \mathrm{Co}$ source are present, each $7.5 \mathrm{~cm}$ from the needle. The resulting spectra are shown

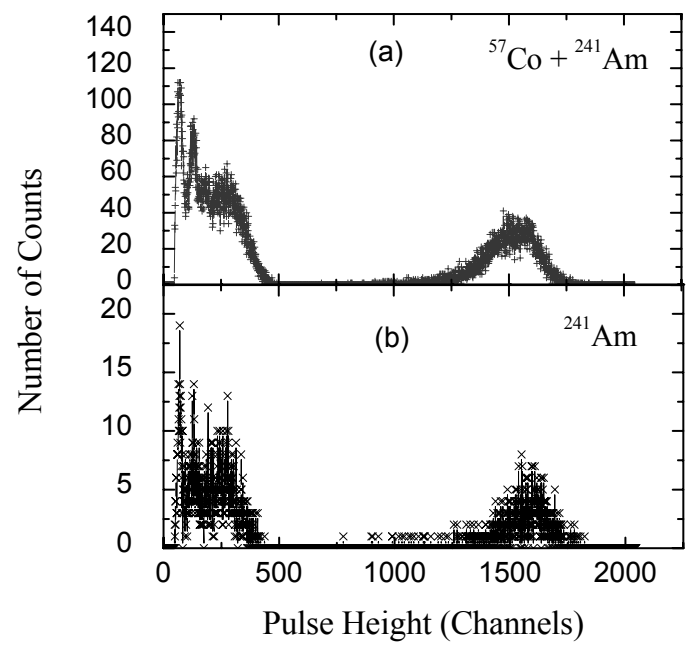

Fig. 4. Pulse height spectrum at $2200 \mathrm{~V}$ in LAr with $250 \mathrm{ppm} \mathrm{Xe,} 5$ psig $\mathrm{He}$ overpressure. The location of the peaks near channel 1500 is pressure dependent. In (a) both the ${ }^{241} \mathrm{Am}$ and ${ }^{57} \mathrm{Co}$ sources are present, and (b) is for the ${ }^{241} \mathrm{Am}$ source alone. The collection times were $240 \mathrm{sec}$ for both (a) and (b). The ${ }^{241} \mathrm{Am}$ source was $15 \mathrm{~cm}$ from the needle tip. in Fig. 4. As can be seen, the rate of pulses induced by the ${ }^{57} \mathrm{Co}$ source plus the ${ }^{241} \mathrm{Am}$ source is approximately five times larger than with the ${ }^{241} \mathrm{Am}$ source alone, consistent with the known intensities and materials in front of the sources. Except for this difference in rate, the pulse height spectra are, nevertheless, essentially identical, confirming our previous observation in the $\mathrm{LAr} / \mathrm{Xe}$ mixtures that the avalanches are saturated, i.e. independent of the incident gamma-ray energy.

\section{Variation of Pulse Height with Gap}

We have tried to verify our analytic calculation of the field, and the threshold voltage for initiating avalanches in $\mathrm{LAr} / \mathrm{Xe}$ by varying the geometry of our detector. As described in [1], the electric field, $\mathrm{E}$ on the tip of the needle in our geometry is given approximately by $\mathrm{E}=\mathrm{VA} /(\mathrm{kTH})$, where $\mathrm{V}, \mathrm{A}$ and $\mathrm{H}$ are defined in Fig. 2. The radius of the needle tip is $\mathrm{T} \sim 0.25 \mu \mathrm{m}$, and $\mathrm{k}=4.4$ is a dimensionless constant that depends logarithmically on the geometry. In Fig. 5 we show the relative pulse height versus voltage for two different values of $\mathrm{H}$, with all other dimensions held approximately constant. We see that for the smaller gap, $\mathrm{H}=$ $4.8 \mathrm{~mm}$, the avalanches are initiated at a voltage of $\mathrm{V} \approx$ $1550 \mathrm{~V}$, whereas for the larger gap of $7.7 \mathrm{~mm}$ the avalanche region starts at $\mathrm{V} \approx 2050 \mathrm{~V}$. The three sets of data for $7.7 \mathrm{~mm}$ were taken on different runs, and differ somewhat due to systematic effects, as described in section II-B. The ratio of these voltages is approximately 0.75 , consistent with that of the gap heights, $4.8 / 7.7=0.62$, within estimated error. This agreement provides some confidence in our description of the electric field.

\section{Avalanche Mode with the Krypton Mixtures}

In the case of pure liquid argon, the avalanche behavior is erratic and unstable, with the pulse amplitude and rate changing over tens of seconds, or disappearing altogether, only to re-appear a few seconds later. The addition of even a

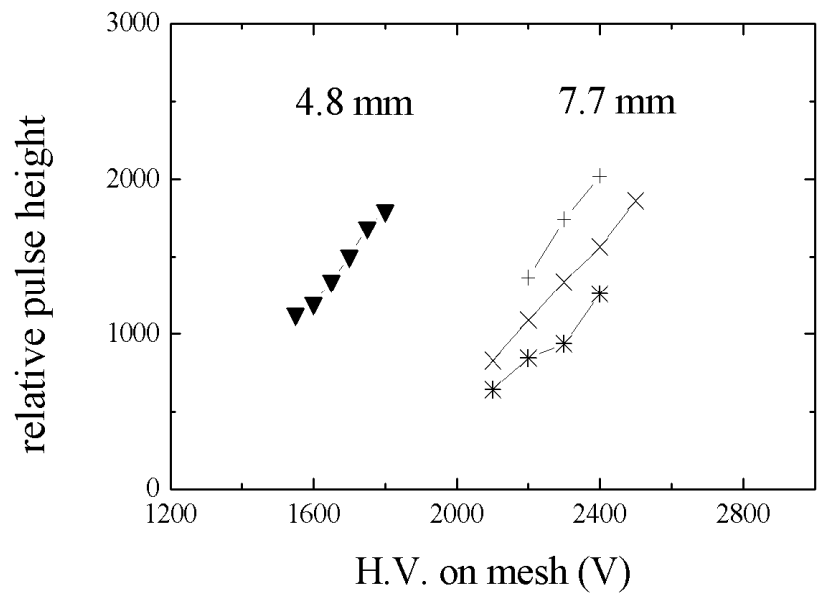

Fig. 5. Mean PH of avalanches versus high voltage, for gaps of $4.8 \mathrm{~mm}$ and $7.7 \mathrm{~mm}$ (dimension $\mathrm{H}$ of fig. 2). Results from three tests with a $7.7 \mathrm{~mm}$ gap are shown (see text). The Xe concentration for all tests is $250 \mathrm{ppm}$. The plot is for the pressure dependent pulses at 5 psig He overpressure. 
small amount of Xe $(\leq 100 \mathrm{ppm})$ stabilized the performance [1]. We also achieved satisfactory results adding tetramethylsilane (TMS) to LAr, but discontinued its use after finding silicon deposits on the needle tip, which we believe came from dissociation of the TMS molecules. In Table I we show the cross sections and rate constants for the disassociation of Ar eximers in gaseous systems [10]. As can be seen, Xe is as effective in disassociating Ar dimers as traditional additives used in proportional chambers such as $\mathrm{CO}_{2}$ or $\mathrm{CH}_{4}$. The energy transfer from LAr to rare gas solutes has been shown to be dominated by collisions with $\mathrm{Ar}$ excitons in the lowest lying $n=1\left({ }^{2} \mathrm{P}_{3 / 2}\right)$ and $n=1\left({ }^{2} \mathrm{P}_{1 / 2}\right)$ states [11]. The ionization energies of $\mathrm{Xe}$ and $\mathrm{Kr}$ dissolved in $\mathrm{LAr}$ are $10.58 \mathrm{eV}$ and $12.5 \mathrm{eV}$, respectively, and the lowest lying $\mathrm{n}=1\left({ }^{2} \mathrm{P}_{3 / 2}\right)$ and $\mathrm{n}=1\left({ }^{2} \mathrm{P}_{1 / 2}\right)$ Ar* states are at $12.0 \mathrm{eV}$ and $12.3 \mathrm{eV}$, respectively [11]. Hence, these Ar excitons can ionize Xe but not $\mathrm{Kr}$. Ionization of $\mathrm{Xe}$ or $\mathrm{Kr}$ by collisions with $\mathrm{Ar}_{2}{ }^{*}$ is also energetically forbidden [11]. Based on these facts, we chose to try $\mathrm{Kr}$ admixtures in $\mathrm{LAr}$, with the expectation that more $\mathrm{Kr}$ than $\mathrm{Xe}$ would be required to stabilize avalanches, as was substantiated.

Table I. Rate constants and cross sections for $\mathrm{Ar}_{2}{ }^{*}+\mathrm{M}->2 \mathrm{Ar}+$ $\mathrm{M}^{*}$ in gases, adapted from [10]

\begin{tabular}{|c|c|c|}
\hline $\begin{array}{c}\text { Acceptor } \\
(\mathrm{M})\end{array}$ & Rate Constant & Cross Section \\
$\left(10^{-11} \mathrm{~cm}^{3} / \mathrm{sec}\right)$ & $\left(10^{-16} \mathrm{~cm}^{2}\right)$ \\
\hline $\mathrm{Kr}$ & 10 & 25 \\
\hline $\mathrm{Xe}$ & 50 & 140 \\
\hline $\mathrm{CH}_{4}$ & 55 & 120 \\
\hline $\mathrm{CO}_{2}$ & 68 & 140 \\
\hline
\end{tabular}

We observed avalanches in mixtures of LAr and small concentrations of Kr. The signal amplitude depended upon voltage and pressure. With the addition of a small concentration of $\mathrm{Kr}$, stable avalanche signals were observed, but only starting at higher concentrations of $\mathrm{Kr}$ than for the case of Xe. For smaller concentrations of $\mathrm{Kr},<1000 \mathrm{ppm}$, the avalanches were erratic and unpredictable, similar to the behavior with pure LAr. The observed pulse height is approximately linearly dependent on the applied HV, but with less dependence on the $\mathrm{Kr}$ concentration (Fig. 6) than in the case of $\mathrm{Xe}[1]$. With $\mathrm{Kr}$ mixtures, we observed only one set of pulses, which were pressure dependent: the amplitude decreasing $\sim 30 \%$ when the gauge pressure was increased from 0 to $1.6 \mathrm{~atm}$. We attribute this behavior to avalanches in gas bubbles formed on the tip of the needle, the same as our interpretation of similar results in $\mathrm{LAr} / \mathrm{Xe}$ mixtures. Unlike the case with $\mathrm{Xe}$, we did not observe pulses that were independent of pressure.

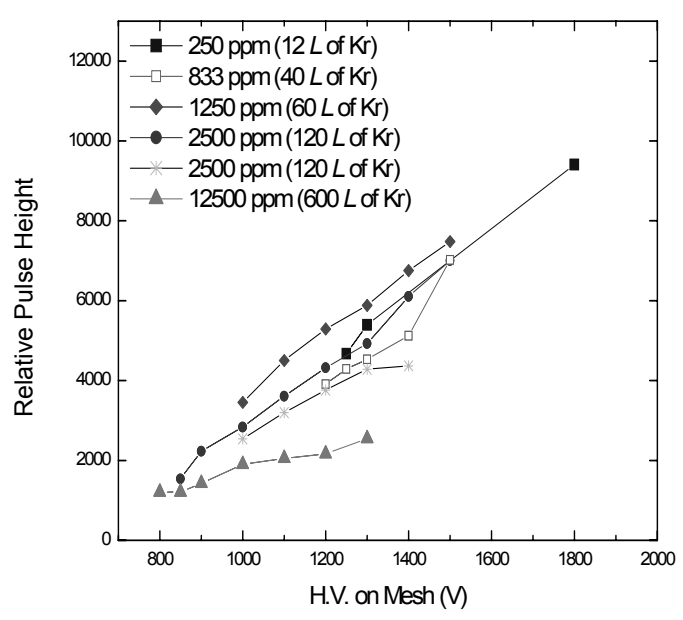

Fig. 6. Pulse height of signals in LAr plus $\mathrm{Kr}$ vs. H.V. for various $\mathrm{Kr}$ concentrations.

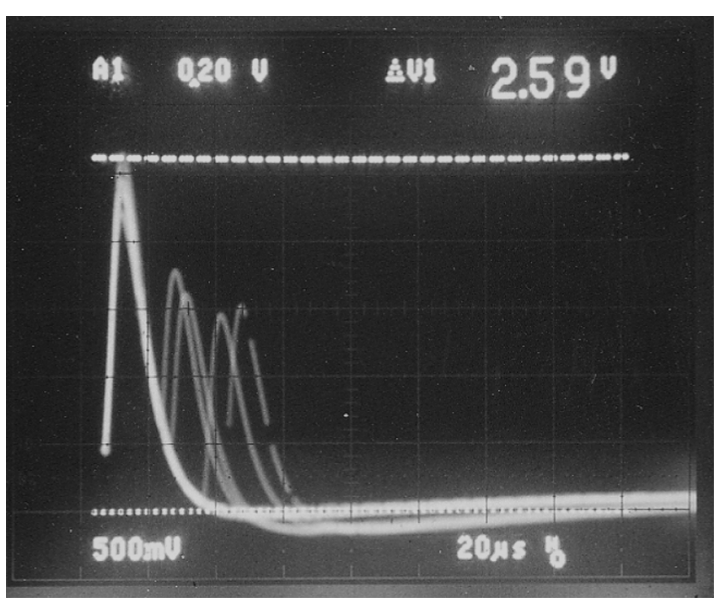

Fig. 7. Photograph of oscilloscope traces of pulses seen in LAr with $2500 \mathrm{ppm} \mathrm{Kr}$ and $1400 \mathrm{~V}$ applied to the drift electrode at $\sim 1.3$ atmospheres pressure (5psig). First (and largest) pulse is about $2.6 \mathrm{~V}$, and the pulse width of $\sim 1 \mu \mathrm{s}$ fwhm is determined by the amplifier time constants.

The pulses in Krypton were typically followed by smaller afterpulses, occurring $\sim 10$ to $100 \mu \mathrm{sec}$ after the original pulse (Fig. 7). The rate of afterpulses decreased with decreasing applied voltage. We did not observe this behavior in LAr with $\mathrm{Xe}$, except at the highest voltage settings.

\section{E. Stirring and Sampling}

We have measured the concentration of Xe dissolved in the liquid by spectrographic analysis [12] and compared the results with the expected concentration based on the amount of Xe gas added to the LAr. We believe this is the first time that this procedure has been followed and reported. For the results reported in [1] we assumed that all the Xe we added to the LAr dissolved in the LAr. For the later tests, we implemented a mixing system using a pivoted alnico bar magnet rotated under the influence of an external driving magnet. Since then we have improved our mixing system and added a sampling system that extracts a $\sim 10 \mathrm{ml}$ sample of the 
LAr/Xe mixture. Then, by heating, the liquid sample is expanded to the gaseous state and into a one- liter sampling bottle that can be shipped to a laboratory for analysis. The results are shown in Fig.8. Several purge-pump cycles of the liquid sampling process were repeated before obtaining a final sample for analysis. By following this procedure, the expected concentration is reasonably well confirmed by the analysis, within about $30 \%$ (Fig. 8). In order to obtain reliable results, we found it essential to have good mixing of the liquid, and believe the major systematic error in this procedure is the extent to which the Xe may be concentrated in the cryo-valve outlet during the pump/purge cycles.

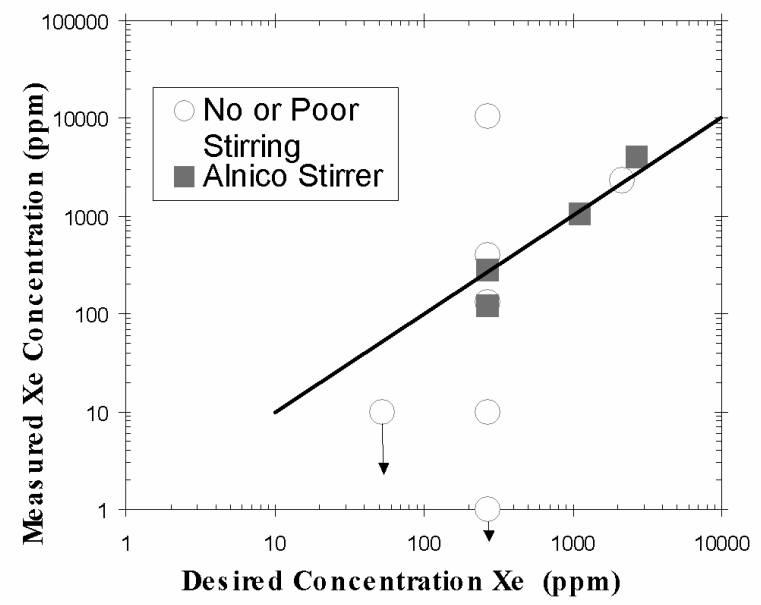

Fig. 8. Stirring and sampling results for Xe in LAr. The diagonal line is not a fit to the data, but indicates where the desired concentration equals the measured concentration of Xe. Downward pointing arrows (without good stirring) indicate the value is an upper limit on the Xe concentration

\section{F. Spindt Cathodes}

We tested an array of $\sim 10,000$ Spindt cathode cells [13], each emitter consisting of a $0.75 \mu \mathrm{m}$ high cone (or "tip") surrounded by a metallic cathode (or "gate") approximately level with the top of the tip (see Fig. 9). The emitter array has a diameter of $\sim 1 \mathrm{~mm}$. The Spindt cathodes were placed in the same plane as our ground electrode, but electrically isolated from it. The distance from the ground plane to the drift electrode was $6 \mathrm{~mm}$, and the drift field was in the range 1000 $\mathrm{V} / \mathrm{cm}$ to $2000 \mathrm{~V} / \mathrm{cm}$. Originally intended for use as a field emission electron source in vacuum, we used the Spindt cathodes in reverse polarity, so that the cone becomes an anode to allow electron avalanche multiplication. As a field emission device, electric fields of $\sim 50 \mathrm{MV} / \mathrm{cm}$ have been achieved on the conical tips at $\sim 120 \mathrm{~V}$ tip-gate voltage, or a factor of seven higher than necessary for avalanching in LAr. We briefly $(\sim 20 \mathrm{sec})$ observed small pulses in LAr/Xe $(250$ $\mathrm{ppm})$ at $\sim 70 \mathrm{~V}$ tip-gate voltage, but these were rapidly followed by breakdown of the device, confirmed via the subsequent large current drawn between the tips and gate at low voltages $(5-12 \mathrm{~V})$. The electrical short between the tips and gate could sometimes be removed by discharging a $1 \mu \mathrm{F}$ capacitor charged to $50 \mathrm{~V}$ across the device. The observed pulses may have been indicators of the incipient breakdown of the device. An alternative explanation is that avalanche multiplication is accompanied by bubble formation at the tip, and the bubble, though very small, creates a conducting path for a spark, resulting in a breakdown or discharge rather than the desired avalanches.

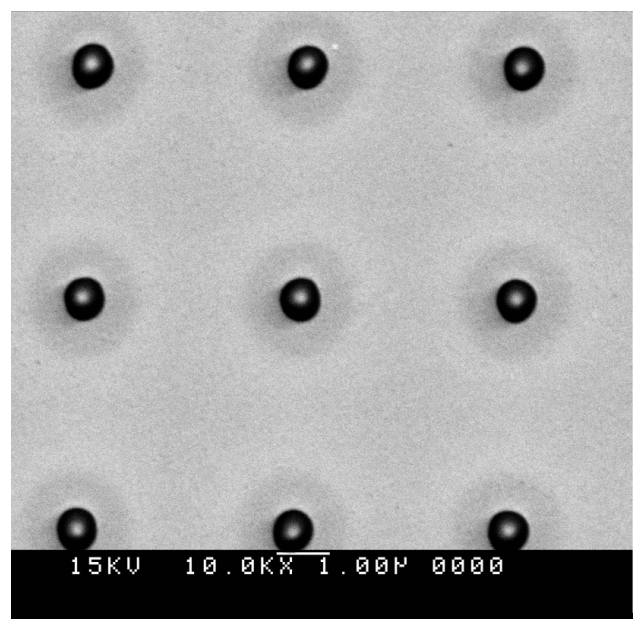

Fig. 9. Micrograph of Spindt cathode surface used in our tests. The diameter of the gates is $\sim 0.75 \mu \mathrm{m}$.

\section{CONCLUSIONS}

In our previous paper [1], small concentrations of Xe were mixed with LAr, in order to stabilize the electron avalanche process, which is otherwise erratic in pure LAr. In the present work, we have analyzed the concentration of Xe actually dissolved in the liquid and compared the results with expectations based on the amount of $\mathrm{Xe}$ gas added to the LAr. There was good agreement between the expected and analyzed concentrations when proper mixing and sampling procedures were followed.

Our measurement of the positive charge carrier mobility in LAr with small admixtures of Xe is $6.4 \times 10^{-3} \mathrm{~cm}^{2} / \mathrm{Vsec}$, in approximate agreement with the mobility measured by others in pure LAr, where the positive charge carriers are principally holes, not positive ions.

In studying avalanche behavior in LAr with small $\mathrm{Kr}$ admixtures we find only pressure-dependent pulses, which we attribute to avalanches forming in gas bubbles. The pulse height increases approximately linearly with applied voltage.

We were unsuccessful in producing avalanches using a Spindt cathode device operated in reverse polarity. An alternative design would be to scale up the cell size by a factor of $\sim 100$, so that small bubbles $(<1 \mu \mathrm{m})$, if present, do not lead to electrical discharges between the tips and gate.

\section{ACKNOWLEDGMENTS}

We thank E. Saiz for help with the electron microscope facility, and professors K. Abe and H. Yoshimoto for supplying samples of TMS and TMG. 


\section{REFERENCES}

[1] J. G. Kim, S. M. Dardin, K.H. Jackson, R. W. Kadel, J. A. Kadyk, V. Peskov, W. A. Wenzel, "Studies of Electron Avalanche Gain in Liquid Argon", IEEE Trans Nucl Sci., vol. 49, no. 4, pp. 1851-1856, Aug, 2002.

[2] ${ }^{57} \mathrm{Co}$ Source purchased from Isotope Products, Inc., Burbank, CA 91504, USA.

[3] Matheson 4A molecular sieve (model 461), Newark, CA 94560, USA.

[4] Messer Oxysorb $\mathrm{O}_{2}$ filter, Allentown, PA 18104, USA.

[5] Gatekeeper inert gas purifier, Aeronex Inc, 6975 Flanders Dr, San Diego, CA 92121, USA.

[6] B. Halpern and R. Gomer, "Field Emission in Liquids", J. of Chem. Phys., vol. 51, no. 3. pp. 1031-1047, Aug. 1969.

[7] K. Arii and W. Schmidt, "Current Injection and Light Emission in Liquid Argon and Xenon in a Divergent Electric Field", IEEE Trans. on Elec. Insul., vol. EI-19, no. 1, pp. 16-23, Feb. 1984.

[8] C. Grun and R. Loveman, "A Review of the Physical Properties of Liquid Ionization Media", IEEE Trans. on Nucl. Sci., vol. NS-26, no. 1, pp. 110-119, Feb. 1979.

[9] O. Hilt and W. Schmidt, "Positive hole mobility in Liquid Xenon", Chem. Phys., vol. 183, pp. 147-153, 1994.

[10] T. Oka, M. Kogoma, M. Imamura, and S. Arai, "Energy transfer of argon excited diatomic molecules", J. Chem. Phys, vol 70, no.7, pp. 3384-3389, Apr. 1979.

[11] S. Kubota, A. Nakamoto, T. Takahashi, S Konni, T. Hamada, M. Miyajima, et al, "Evidence of the existence of exciton states in liquid argon and exciton-enhanced ionization from xenon doping", Phys. Rev. $B$, vol. 13, no. 4, pp. 1649-1653, Feb. 1976.

[12] Atlantic Analytical Laboratory, Inc., P. O. Box 220, Salem Industrial Park, Building 44, Whitehorse, NJ 08888, USA.

[13] Provided by, and the intellectual property of SRI, contact Dr. Capp Spindt, Director, Vacuum Microelectronics Program, Applied Physics Sciences Laboratory, SRI, 333 Ravenswood Ave, Menlo Park, CA 94025, USA. 